\title{
Eicosanoid/thromboxane $A_{2}$-independent and -dependent generation of Iysoplasmenylethanolamine via phospholipase $A_{2}$ in collagen-stimulated human platelets
}

\author{
Marco E. TURINI and Bruce J. HOLUB* \\ Department of Nutritional Sciences, University of Guelph, Guelph, Ontario, Canada N1G 2W1
}

Collagen-induced human platelet stimulation is dependent on the release of arachidonic acid (AA) from membrane phospholipid and the formation of thromboxane $A_{2}\left(T x A_{2}\right)$ for $\mathrm{TxA}_{2}$-induced platelet activation. Since plasmenylethanolamine represents the single major phospholipid reservoir of $\mathrm{AA}$ in resting human platelets, we assessed its hydrolysis via phospholipase $\mathrm{A}_{2}$ upon platelet stimulation with low levels of collagen by determining the generation of $\left[{ }^{3} \mathrm{H}\right]$ lysoplasmenylethanolamine via eicosanoid/TxA $\mathrm{T}_{2}$-independent and -dependent processes. Ethanolamine phospholipids in platelets were prelabelled with $\left[{ }^{3} \mathrm{H}\right]$ ethanolamine before stimulation with either collagen or the $\mathrm{TxA}_{2}$ mimetic $\mathrm{U} 46619$, in the presence or absence of $\mathrm{BW} 755 \mathrm{C}$, a dual inhibitor of the cyclooxygenase/lipoxygenase activities, or GR32191B, a TxA receptor antagonist. Collagen stimulation promoted a marked generation of $\left[{ }^{3} \mathrm{H}\right]$ lysoplasmenylethanolamine, which was only moderately decreased when $\mathrm{TxA}_{2}$ synthesis or $\mathrm{TxA}_{2}$ receptors were blocked by BW755C or GR32191B respectively. The moderate rise in $\left[{ }^{3} \mathrm{H}\right]$ lysoplasmenylethanolamine formation with $\mathrm{U} 46619$ as the agonist was only slightly affected by BW755C and blocked by GR32191B. Evidence for eicosanoid/TxA independent and -dependent generation of $\left[{ }^{3} \mathrm{H}\right]$ lysophosphatidylethanolamine was also obtained. A significant quantitative loss of AA from plasmenylethanolamine was also demonstrated in collagen-stimulated platelets. The present findings indicate the activation of plasmenylethanolamine cleavage via phospholipase $A_{2}$ in collagen-stimulated human platelets, which, to a considerable extent, does not depend on eicosanoid/ $\mathrm{TxA}_{2}$ synthesis. This may represent an important source of releasable $\mathrm{AA}$ for $\mathrm{TxA}_{2}$ generation and the promotion of further liberation of $\mathrm{AA}$ and phospholipid-mediated signalling pathways.

\section{INTRODUCTION}

Platelet stimulation by agonists such as collagen, thrombin or the calcium ionophore A23187 leads to the release of arachidonic acid (AA; $\mathrm{C}_{20: 4, n-6}$ ) from membrane phospholipids [1-6]. The stimulated release of AA upon receptor occupancy, via phospholipase $\mathrm{A}_{2}$, and the subsequent metabolism of AA through the cyclooxygenase and thromboxane synthetase generates the potent pro-aggregatory metabolite thromboxane $A_{2}\left(T_{x} A_{2}\right)$. Recent studies in human platelets have focused on AA turnover within the individual phospholipids [2,4,5,7-11]. Much of the research in this area has employed high levels of thrombin as an agonist which permits the monitoring of radioactivity loss from the various phospholipids after pre-labelling with AA. The latter studies have indicated that phosphatidylcholine [1,2,4,5,7,9-11] and phosphatidylethanolamine [5,9] are major sources of phospholipase $\mathrm{A}_{2}$-mediated AA release, whereas some AA can also be freed by diacylglycerol/monoacylglycerol lipase activities after phospholipase $C$ action on phosphoinositides $[12,13]$. The possible role of plasmenylethanolamine (1-O-alk-1'-enyl-2-acyl$s n$-glycero-3-phosphoethanolamine) as a source of AA for $\mathrm{TxA}_{2}$ synthesis has also been suggested $[2,7,10,11]$.

Collagen-induced platelet aggregation is dependent on the early release of $\mathrm{AA}$ and its subsequent conversion into $\mathrm{TxA}_{2}$ [14-18], especially at lower levels of collagen $[16,18]$. $\mathrm{TxA}_{2}$ can then further promote phospholipase $\mathrm{A}_{2}$-mediated release of $\mathrm{AA}$ for eicosanoid synthesis, phosphoinositide turnover and trans- membrane signalling $[6,19]$. The release of AA via phospholipase $A_{2}$ is accompanied by the liberation of the corresponding 1-acyl2-lyso-phospholipids [20]. At lower collagen levels, it has been possible to monitor the individual $\left[{ }^{14} \mathrm{C}\right]$ stearoyl-labelled 1-acyl-2lyso-phospholipids generated via phospholipase $A_{2}$ activation under conditions where no marked decrease in the corresponding parent phospholipids was readily observed [21]. Such studies indicate that the diacylphospholipids, namely phosphatidylcholine, phosphatidylethanolamine, phosphatidylserine and phosphatidylinositol, can be degraded by a combination of eicosanoid-independent and -dependent processes, the $\mathrm{TxA}_{2}$ dependent pathway being particularly active on phosphatidylcholine.

It has been determined that $45-60 \%$ of the ethanolaminephospholipid in human platelets is represented by plasmenylethanolamine [22-24]. Since $68-72 \%$ of the fatty acids residing in the $s n-2$ position of the plasmenylethanolamine is represented by AA $[22,25]$, this specific phospholipid is a significant contributor to the total AA-containing phospholipid in human platelets. It can be calculated that plasmenylethanolamine represents $30 \%$ of the total reservoir of AA-containing phospholipid in resting human platelets, whereas the phosphatidylcholine and phosphatidylethanolamine account for $21 \%$ and $16 \%$ respectively $[22,26]$.

In view of the essential role of phospholipase $A_{2}$ activation and subsequent $\mathrm{TxA}_{2}$ synthesis for platelet activation by collagen, as well as the predominance of AA-containing plasmenyl-

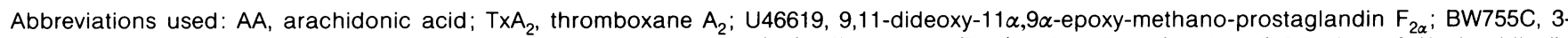
amino-1-(3-trifluoromethylphenyl)-2-pyrazoline; GR32191B, $\{1 R-[1 \alpha(Z), 2 \beta, 3 \beta, 5 \alpha]\}-(+)-7-\left(5-\left\{\left[1,1^{\prime}\right.\right.\right.$-biphenyl)-4-yl]methoxyl\}-3-hydroxy-2-(1-piperidinyl)cyclopentyl)-4-heptenoic acid hydrochloride; ANS, 8-anilinonaphthalene-1-sulphonic acid; GPE, sn-glycero-3-phosphoethanolamine.

* To whom correspondence should be addressed. 
ethanolamine in human platelets, it was decided to study the eicosanoid/Tx $\mathrm{A}_{2}$-independent and -dependent formation of lysoplasmenylethanolamine (1-O-alk-1'-enyl-2-lyso-sn-glycero3-phosphoethanolamine) in collagen-stimulated platelets. For this purpose, ethanolamine phospholipids in platelets were prelabelled with $\left[{ }^{3} \mathrm{H}\right]$ ethanolamine before stimulation with either collagen or the $\mathrm{TxA}_{2}$ mimetic, $\mathrm{U} 46619$, in the presence and absence of either BW755C or GR32191B.

The present findings indicate that a significant portion of the lysoplasmenylethanolamine generation, resulting from the action of phospholipase $\mathrm{A}_{2}$ on plasmenylethanolamine, occurred in an eicosanoid/TxA $\mathrm{A}_{2}$-independent manner.

\section{MATERIALS AND METHODS}

\section{Materials}

2-Amino $\left[1-{ }^{3} \mathrm{H}\right]$ ethanol hydrochloride $(29.5 \mathrm{Ci} / \mathrm{mmol})$ in aqueous solution was purchased from Amersham Canada Ltd. (Oakville, Ont., Canada). The $\left[{ }^{3} \mathrm{H}\right] \mathrm{TxB}_{2}$ radioimmunoassay kit was obtained from Du Pont-New England Nuclear (Lachine, PQ, Canada). Pre-coated t.l.c. plates (silica gel 60) were from E. Merck, Associate of BDH Chemicals Canada (Toronto, ON, Canada). Collagen was from Hormon-Chemie (München, Germany), and U46619 was supplied by Upjohn Co. (Kalamazoo, MI, U.S.A.) as a methyl acetate solution which was evaporated under nitrogen, dissolved in ethanol, and stored below $0{ }^{\circ} \mathrm{C}$ before dilution with saline. BW755C was provided by Dr. R. Ippersiel of the Wellcome Research Laboratories (Kirkland, PQ, Canada). GR32191B was a gift from Dr. B. M. Bain of the Glaxo Group Research Ltd. (Greenford, Middx., U.K.). Lysophosphatidylethanolamine (1-acyl-2-lyso-sn-glycero-3phosphoethanolamine), and a standard mixture of plasmenylethanolamine and (diacyl)phosphatidylethanolamine, as well as 8-anilinonaphthalene-1-sulphonic acid (ANS), were from Sigma Chemical Co. (St. Louis, MO, U.S.A.). The liquid-scintillation solution CytoScint was from ICN Biomedicals (Irvine, CA, U.S.A.). All chemicals and solvents were of analytical grade.

\section{Platelet isolation and pre-labelling}

Blood was obtained by antecubital venepuncture from healthy male volunteers between 24 and 30 years of age who denied taking any medication for at least 10 days and alcohol for 2 days before the experiments. Washed platelet suspensions were prepared by the method of Mustard et al. [27], except that Hepes ( $5 \mathrm{mM}$ final concn.) was added to the modified Tyrodes buffer. Platelets were pre-labelled in a metabolic-shaker waterbath at $37^{\circ} \mathrm{C}$ for $2 \mathrm{~h}$ with $\left[{ }^{3} \mathrm{H}\right]$ ethanolamine $(0.5 \mathrm{mCi} / 50-60 \mathrm{ml}$ initial blood vol.) in the first washed platelet suspension $(5 \mathrm{ml})$. Platelet counts were determined with a Coulter Counter model ZM (Coulter Electronics of Canada Ltd., Burlington, Ont., Canada) and adjusted to a final concentration of $5 \times 10^{8}$ platelets $/ \mathrm{ml}$. This labelling procedure gave mean d.p.m. (all experiments) of $165 \times 10^{3}$ and $379 \times 10^{3}$ in the plasmenylethanolamine and phosphatidylethanolamine, respectively, per lipid extract employed for t.l.c. separation. No statistically significant alteration in the radioactivity associated with the parent phospholipids was observed $(P>0.05)$ on platelet stimulation (results not shown).

\section{Platelet incubations}

Samples $(1 \mathrm{ml})$ of platelet suspensions were preincubated for $1 \mathrm{~min}$ in silicone-treated cuvettes with stirring at $900 \mathrm{rev} . / \mathrm{min}$, $37^{\circ} \mathrm{C}$, in an aggregometer (Payton Associates, Buffalo, NY, U.S.A.). Then $10 \mu \mathrm{l}$ of the dual cyclo-oxygenase/lipoxygenase inhibitor [28] BW755C (100 $\mu \mathrm{M}$ final conen.), the $\mathrm{TxA}_{2}$-receptor antagonist [29] GR32191B (1 $\mu \mathrm{M}$ final concn.), or the vehicle, water, was added $2 \mathrm{~min}$ before platelet stimulation with either collagen $(20 \mu \mathrm{l} ; 2 \mu \mathrm{g} / \mathrm{ml}$ final concn.) or U46619 $(10 \mu \mathrm{l} ; 1 \mu \mathrm{M})$. The reactions were stopped after 2 min stimulation by the rapid addition of $3.75 \mathrm{ml}$ of chloroform/methanol ( $1: 2$, by vol.), and the lipids extracted.

\section{Lipid extraction and phospholipid analyses}

Lipids were extracted by the method of Bligh and Dyer [30], except that $1.25 \mathrm{ml}$ of $1.8 \% \mathrm{KCl}$ solution was used instead of water. The chloroform phase was washed with $4.75 \mathrm{ml}$ of methanol $/ 0.9 \% \mathrm{KCl}(10: 9$, by vol.), and lipids from the initial methanol $/ 0.9 \% \mathrm{KCl}$ upper phase were re-extracted with $2.5 \mathrm{ml}$ of chloroform. The chloroform phases were pooled, dried under nitrogen at $40^{\circ} \mathrm{C}$, and resuspended in $25 \mu \mathrm{l}$ of chloroform/methanol (2:1, by vol.). Phospholipids were separated by two-dimensional t.l.c. by a modification of the method of Horrocks [31]. Phospholipid samples, to which $40 \mu \mathrm{g}$ of unlabelled lysophosphatidylethanolamine standard was added, were spotted in the lower right-hand corner of silica-gel 60 plates, previously activated for $30 \mathrm{~min}$ at $80^{\circ} \mathrm{C}$. The plates were developed for $90 \mathrm{~min}$ in a paper-lined chromatographic tank, which was pre-equilibrated for 45-60 min with the first solvent consisting of chloroform/methanol/14.8 $\mathrm{M} \mathrm{NH}$ (130:70:11, by vol.). This first development yielded a mixed fraction containing phosphatidylethanolamine plus plasmenylethanolamine and another containing lysophosphatidylethanolamine plus lysoplasmenylethanolamine (resulting from phospholipase $\mathrm{A}_{2}$ cleavage of plasmenylethanolamine in stimulated platelets). Between dimensions, the plates were removed, dried for $10 \mathrm{~min}$ at ambient temperature and then dried in an oven at $40^{\circ} \mathrm{C}$ for $30 \mathrm{~min}$. A $4 \mathrm{~cm}$ band of the silica-gel plate, containing the mixed parent and mixed lyso forms of ethanolamine-containing phospholipid, was exposed for $3 \mathrm{~min}$ to conc. $\mathrm{HCl}$ fumes by facing the plate over a Pyrex oven dish $(23 \mathrm{~cm} \times 14 \mathrm{~cm})$ containing $100 \mathrm{ml}$ of $\mathrm{HCl}$. The plates were dried again at $40^{\circ} \mathrm{C}$ for $15 \mathrm{~min}$, before being developed from the right edge for $2 \mathrm{~h}$ in the second solvent system containing chloroform $/$ methanol $/ 88 \%$ formic acid/water (55:28:5:1, by vol.). Individual phospholipids were observed under u.v. light after spraying with $0.1 \%$ ANS solution in water [32]. The areas containing phosphatidylethanolamine, lyso(2-acyl)phosphatidylethanolamine (generated by $\mathrm{HCl}$-fume exposure from plasmenylethanolamine), lysophosphatidylethanolamine, as well as GPE (generated by $\mathrm{HCl}$ fumes from lysoplasmenylethanolamine) were scraped into scintillation-counting vials. Both ethanolamine-containing lysophospholipids co-migrate during the first t.l.c. development; although not detected by ANS, GPE, which does not migrate during the second t.l.c. development, resides at the origin. Distilled water $(1.5 \mathrm{ml})$ was added to the vials before addition of $16 \mathrm{ml}$ of CytoScint, and the samples were counted for radioactivity in a liquid-scintillation counter. The measured radioactivity in the GPE fraction derived from stimulated minus control platelets represents the $\left[{ }^{3} \mathrm{H}\right]$ lysoplasmenylethanolamine resulting from phospholipase $A_{2}$ (agonist-induced) cleavage.

\section{$\mathrm{TxA}_{2}$ measurement}

Samples $(1 \mathrm{ml})$ of platelet suspensions were stimulated with collagen in the absence and presence of BW755C as described. 
The reactions were stopped by addition of $25 \mu \mathrm{M}$ indomethacin, and the cuvettes were transferred on to ice. Platelet samples were centrifuged at $3000 \mathrm{~g}$ for $15 \mathrm{~min}$ at $4{ }^{\circ} \mathrm{C}$, and the supernatants were removed and stored at $-80^{\circ} \mathrm{C}$. Thromboxane $\mathrm{B}_{2}$, the stable metabolite of $\mathrm{TxA}_{2}$, was determined in duplicate by radioimmunoassay [33].

\section{Mass determination of AA in ethanolamine-containing phospholipids}

The AA content of the plasmenylethanolamine and phosphatidylethanolamine of resting and collagen-stimulated platelets $\left(2 \mu \mathrm{g} / 5 \times 10^{8}\right.$ platelets; $\left.10 \mathrm{~min}\right)$ was determined by procedures similar to those previously described [25,34]. For this purpose, after t.l.c. as described above, the fatty acid methyl esters derived from the lyso(2-acyl)phosphatidylethanolamine, representing the 2-position of the parent plasmenylethanolamine, and from the phosphatidylethanolamine were analysed by g.l.c. in the presence of a known amount of pentadecanoate as an internal standard $[25,34]$.

\section{Statistical analysis}

The data were log-transformed and analysed by analysis of variance (ANOVA). The log-transformation was not performed when ratios were to be analysed. Specific differences for preplanned comparisons between treatment means were examined by Student's $t$ test. The data of AA mass from ethanolaminecontaining phospholipids were analysed by paired $t$ test [35].

\section{RESULTS}

To determine the optimal time of $\mathrm{HCl}$-fume exposure for complete hydrolysis of the alkenyl linkage in the plasmalogenic phospholipids (without detectable cleavage of the acyl forms), preliminary experiments were performed with a standard mixture of plasmenylethanolamine and diacylphosphatidylethanolamine from bovine brain. Maximum hydrolysis of plasmenylethanolamine occurred after $2.5 \mathrm{~min}$, whereas phosphatidylethanolamine remained stable for at least $5 \mathrm{~min}$ upon $\mathrm{HCl}$-fume treatment (results not shown). From these experiments, $\mathrm{HCl}$-fume exposure was routinely set at $3 \mathrm{~min}$. El Tamer et al. [36] reported some acyl-bond hydrolysis by this treatment as compared with phospholipase $\mathbf{A}_{1}$ digestion. However, the acyl-bond hydrolysis observed in their study may be the result of a longer $\mathrm{HCl}$-fume treatment $(10 \mathrm{~min})$.

Measurements of $\mathrm{TxA}_{2}$ in resting and stimulated platelets exposed to collagen for $2 \mathrm{~min}$ gave levels of 2.8 and $69.4 \mathrm{ng} / 5 \times 10^{8}$ platelets respectively, in the absence of BW755C. It was observed that more than $95 \%$ of the agonist-induced formation of $\mathrm{TxA}_{2}$ was blocked by preincubation with $100 \mu \mathrm{M}$ BW755C.

The time-dependent generation of $\left[{ }^{3} \mathrm{H}\right]$ lysoplasmenylethanolamine, via phospholipase $\mathrm{A}_{2}$ action on $\left[{ }^{3} \mathrm{H}\right]$ plasmenylethanolamine, in collagen-stimulated platelets as studied in the absence and presence of BW755C is presented in Figure 1. The corresponding data for $\left[{ }^{3} \mathrm{H}\right]$ lysophosphatidylethanolamine generation from $\left[{ }^{3} \mathrm{H}\right]$ phosphatidylethanolamine hydrolysis are given in Figure 2. Both lysophospholipids exhibited maximal accumulation by 2 min after collagen stimulation. Preincubation of platelets with $100 \mu \mathrm{M}$ BW755C for $2 \mathrm{~min}$ before collagen stimulation only moderately decreased the formation of lysoplasmenylethanotamine (Figure 1) and lysophosphatidylethanolamine (Figure 2). Since a small decrease in the amount of accumulated lyso- phospholipids was obtained with stimulation times extended to $3 \mathrm{~min}$, routine experiments employed exposure periods of $2 \mathrm{~min}$.

The combined data in Table 1 from multiple experiments show that a marked rise in $\left[{ }^{3} \mathrm{H}\right]$ lysoplasmenylethanolamine $(302 \%$ of control values) occurred upon platelet stimulation with collagen. This accumulation upon agonist exposure was only moderately decreased (to $245 \%$ of control values) when $\mathrm{TxA}_{2}$ synthesis was blocked by the addition of BW755C before collagen stimulation. The corresponding values for $\left[{ }^{3} \mathrm{H}\right]$ lysophosphatidylethanolamine

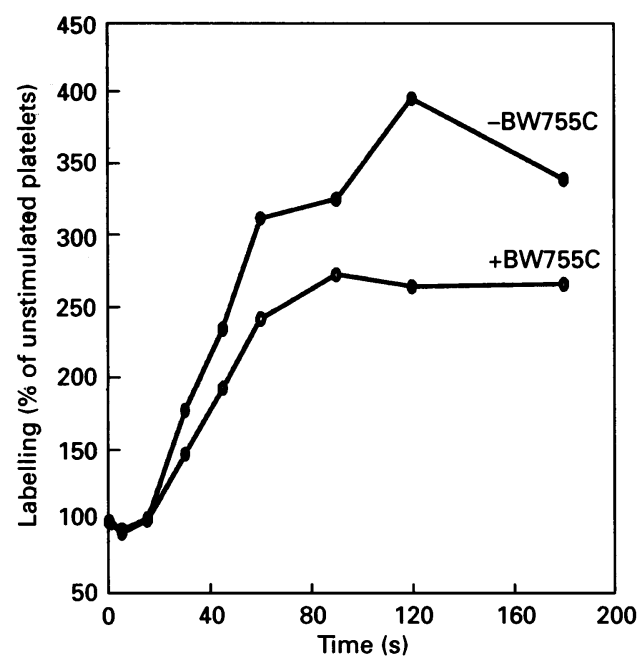

Figure 1 Time course showing generation of radiolabelled lysoplasmenylethanolamine in collagen-stimulated human platelets and effect of BW755C

Platelets were pre-labelled with $\left[{ }^{3} \mathrm{H}\right]$ ethanolamine and subsequently incubated (in the presence and absence of $100 \mu \mathrm{M} \mathrm{BW755C}$ ) for various periods of time with collagen ( $2 \mu \mathrm{g} / \mathrm{ml}$ of platelet suspension). The radioactivity associated with the lysoplasmenylethanolamine was determined after lipid extraction and t:I.c. as described in the Materials and methods section. The data are representative (mean values) of two separate experiments. The mean radioactivity (d.p.m.) in the lysophospholipid of the unstimulated platelets was 579 and 653 in the presence and absence of BW755C respectively.

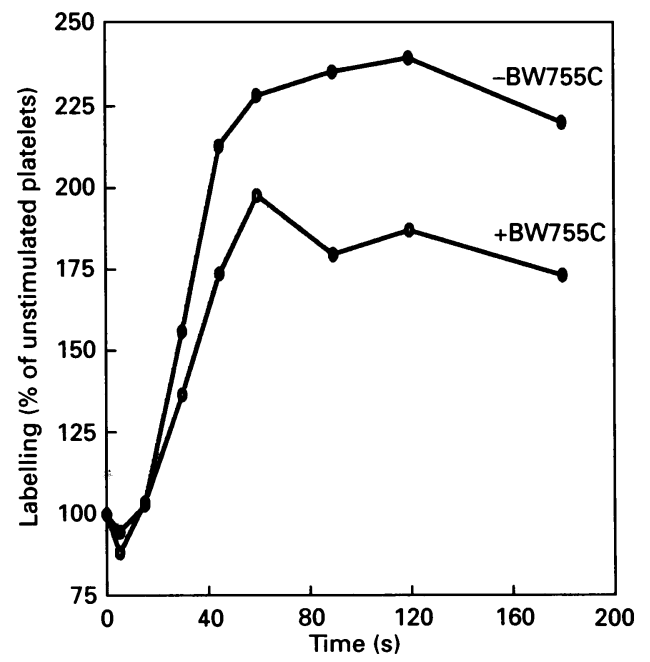

Figure 2 Time course showing generation of radiolabelled Iysophosphatidylethanolamine in collagen-stimulated human platelets and effect of BW755C

Legend as for Figure 1. The mean radioactivity (d.p.m.) in the lysophospholipid of the unstimulated platelets was 2151 and 2507 in the presence and absence of BW755C respectively. 


\section{Table 1 Activation of lysophospholipid generation upon platelet stimulation with collagen or U46619 in the presence and absence of BW755C}

Human platelets were pre-labelled with $\left[{ }^{3} \mathrm{H}\right]$ ethanolamine and subsequently stimulated for 2 min with $2 \mu \mathrm{g}$ of collagen or $1 \mathrm{nmol}$ of U46619 per $\mathrm{ml}$ of platelet suspension (in the presence and absence of $100 \mu \mathrm{M} \mathrm{BW755C)}$. The radioactivity associated with the lysophospholipids (lysoplasmenylethanolamine and lysophosphatidylethanolamine) was determined after lipid extraction and t.I.c. as described in the Materials and methods section. The data are given as mean \pm S.E.M. from separate experiments on 4 subjects (duplicate determinations on each subject). ${ }^{a-c}$ Significantly different $\left({ }^{\mathrm{a}} P<0.05 ;{ }^{b} P<0.01 ;{ }^{c} P<0.0001\right)$ from respective controls (without BW755C) by Student's $t$ test after ANOVA analysis. "Significantly different $(P<0.0001)$ from respective unstimulated controls by Student's $t$ test after ANOVA analysis

\begin{tabular}{|c|c|c|c|c|c|c|}
\hline \multirow[b]{3}{*}{ Lysophospholipid } & \multirow{2}{*}{\multicolumn{2}{|c|}{$\begin{array}{l}\text { Radioactivity } \\
\text { in unstimulated (d.p.m.) }\end{array}$}} & \multicolumn{4}{|c|}{ Stimulated ( $\%$ of unstimulated values) } \\
\hline & & & \multicolumn{2}{|l|}{ Collagen } & \multicolumn{2}{|l|}{ U46619 } \\
\hline & $-B W 755 C$ & $+B W 755 C$ & $-B W 755 C$ & $+B W 755 C$ & $-B W 755 C$ & $+B W 755 C$ \\
\hline $\begin{array}{l}\text { Lysoplasmenylethanolamine } \\
\text { Lysophosphatidyiethanolamine }\end{array}$ & $\begin{array}{c}553 \pm 52 \\
1729+227\end{array}$ & $\begin{array}{r}516 \pm 49^{a} \\
1651 \pm 233\end{array}$ & $\begin{array}{l}302 \pm 4^{d} \\
199 \pm 9^{d}\end{array}$ & $\begin{array}{l}245 \pm 7^{c . d} \\
151 \pm 10^{b . d}\end{array}$ & $\begin{array}{l}132 \pm 2^{d} \\
135 \pm 4^{d}\end{array}$ & $\begin{array}{l}129 \pm 4^{d} \\
124 \pm 9^{d}\end{array}$ \\
\hline
\end{tabular}

Table 2 Comparison of radioactivity ratios (acyl species/1-alkenyl) in lysophospholipids arising from agonist exposure relative to corresponding parent phospholipids

The ratios of the net radioactivity accumulated (stimulated minus resting platelets) in the lysophospholipids due to agonist addition are given and compared with the corresponding parent phospholipids of the unstimulated platelets. The data are given as mean \pm S.E.M. from separate experiments on 4 subjects (duplicate determinations on each subject). Significance by two-way analysis of variance is indicated. NS, not significantly different

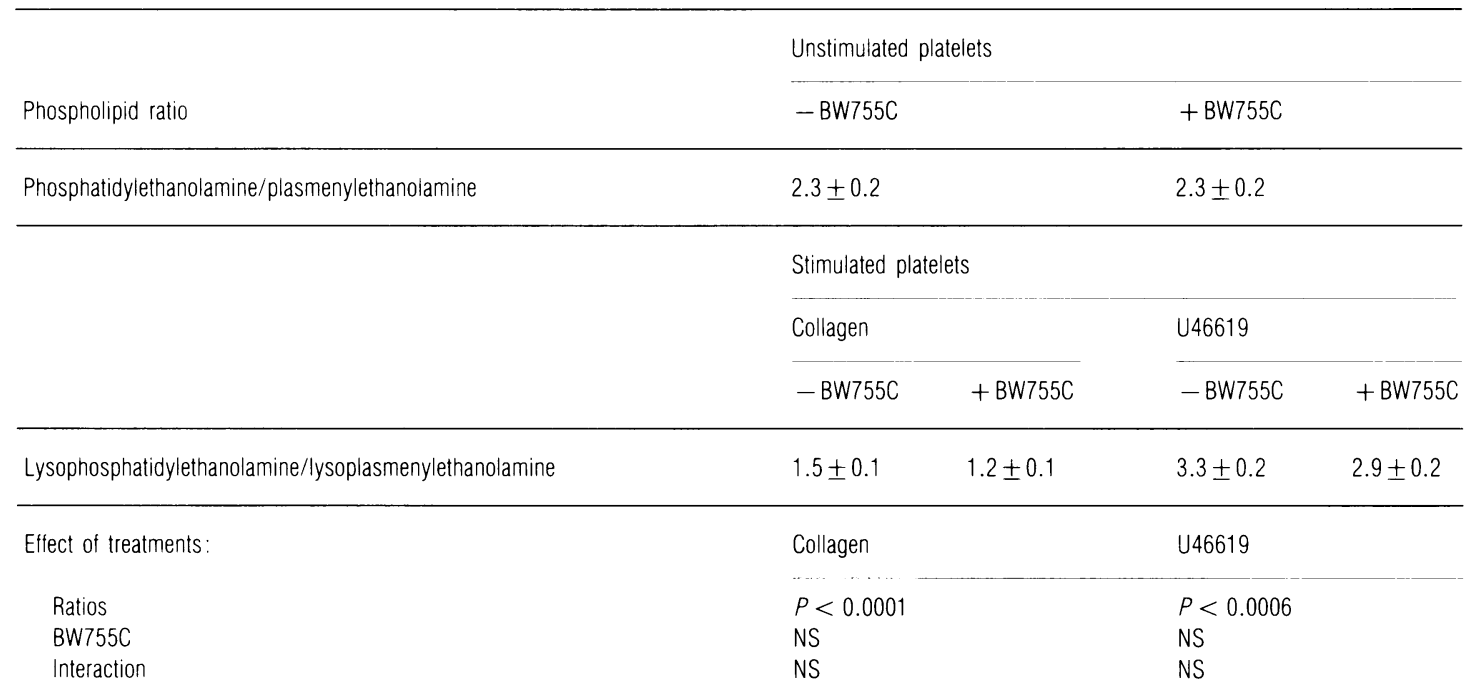

Table 3 Activation of lysophospholipid generation upon platelet stimulation with collagen or U46619 in the presence and absence of GR32191B

Human platelets were pre-labelled with $\left[{ }^{3} \mathrm{H}\right]$ ethanolamine and subsequently stimulated for 2 min with $2 \mu \mathrm{g}$ of collagen or $1 \mathrm{nmol}$ of $\mathrm{U} 46619$ per ml of platelet suspension (in the presence and absence of $1 \mu \mathrm{M}$ GR32191B). The radioactivity associated with the Iysophospholipids (lysoplasmenylethanolamine and lysophosphatidylethanolamine) was determined after lipid extraction and t.l.c. as

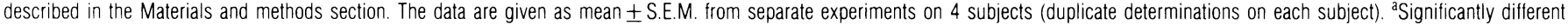
$(P<0.05)$ from respective controls $(-$ GR32191B) by Student's $t$ test after ANOVA analysis. 'Significantly different $(P<0.0001)$ from respective unstimulated controls by Student's $t$ test after ANOVA analysis.

\begin{tabular}{|c|c|c|c|c|c|c|}
\hline \multirow[b]{3}{*}{ Lysophospholipid } & \multirow{2}{*}{\multicolumn{2}{|c|}{$\begin{array}{l}\text { Radioactivity } \\
\text { in unstimulated (d.p.m.) }\end{array}$}} & \multicolumn{4}{|c|}{ Stimulated ( $\%$ of unstimulated values) } \\
\hline & & & \multicolumn{2}{|l|}{ Collagen } & \multicolumn{2}{|l|}{ U46619 } \\
\hline & - GR32191B & + GR32191B & -GR32191B & + GR32191B & - GR32191B & + GR32191B \\
\hline Lysoplasmenylethanolamine & $1127 \pm 159$ & $1033 \pm 110$ & $336 \pm 38^{b}$ & $234 \pm 15^{a, b}$ & $142 \pm 6^{b}$ & $104 \pm 5^{\mathrm{a}}$ \\
\hline Lysophosphatidylethanolamine & $3542 \pm 518$ & $3424 \pm 543$ & $200 \pm 20^{\mathrm{b}}$ & $159 \pm 9^{b}$ & $150 \pm 10^{\mathrm{b}}$ & $111 \pm 7^{\mathrm{a}}$ \\
\hline
\end{tabular}

accumulation resulting from collagen activation were $199^{\circ} \circ$ $(-\mathrm{BW} 755 \mathrm{C})$ and $151^{\circ}{ }_{0}(+\mathrm{BW} 755 \mathrm{C})$ of control values respectively (Table 1). A moderate rise to only $32^{\circ}{ }_{0}$ above control values in $\left[{ }^{3} \mathrm{H}\right]$ lysoplasmenylethanolamine formation was obtained with U46619 as the agonist; this was only slightly affected by preincubation with BW755C (Table 1). The moderate 
Table 4 Loss of AA from ethanolamine-containing phospholipids upon platelet stimulation with collagen

Human platelets were stimulated with collagen, and the mass determinations of $A A$ in the plasmenylethanolamine and phosphatidylethanolamine were performed by combined t.l.c. plus g.I.c. analyses as described in the Materials and methods section. The data are given as means \pm S.E.M. for $A A$ amounts in the ethanolamine-containing phospholipids from separate experiments on 4 subjects (duplicate determinations on each subject). ${ }^{\text {a Significantly different }}$ $(P<0.05)$ from unstimulated control platelets by paired $t$ test.

\begin{tabular}{lll}
\hline Phospholipid & $\begin{array}{l}\text { Unstimulated } \\
\left(\mathrm{nmol} / 5 \times 10^{8} \text { platelets }\right)\end{array}$ & $\begin{array}{l}\text { Stimulated } \\
(\% \text { of unstimulated })\end{array}$ \\
\hline Plasmenylethanolamine & $17.3 \pm 0.5$ & $95.8 \pm 1.0^{\mathrm{a}}$ \\
Phosphatidylethanolamine & $11.7 \pm 0.4$ & $93.5 \pm 1.7^{\mathrm{a}}$
\end{tabular}

increase in $\left[{ }^{3} \mathrm{H}\right]$ lysophosphatidylethanolamine with U46619 to $35 \%$ above control values was also little affected by BW755C (Table 1).

Determination of the ratios for $\left[{ }^{3} \mathrm{H}\right]$ lysophosphatidylethanolamine $/\left[{ }^{3} \mathrm{H}\right]$ lysoplasmenylethanolamine which resulted from collagen stimulation (agonist-induced) gave a mean value of 1.5 (in the absence of BW755C), which was lower than that in the corresponding parent phospholipids (2.3) of resting platelets (Table 2). The lysophospholipid ratio was even smaller in the presence of BW755C (1.2), and approximately half that of the corresponding parent phospholipids. The use of U46619 alone as an agonist gave a corresponding ratio in lysophospholipid of 3.3; this value was not significantly affected by preincubation with BW755C.

Similar results to those observed with BW755C were obtained in collagen-stimulated platelets using the $\mathbf{T x A}_{2}$-receptor antagonist GR32191B (Table 3). A 325\% and 234\% rise in $\left[{ }^{3} \mathrm{H}\right]$ lysoplasmenylethanolamine, and a $200 \%$ and $159 \%$ rise in $\left[{ }^{3} \mathrm{H}\right]$ lysophosphatidylethanolamine, above control values was observed in the presence or absence of GR32191B respectively. A moderate increase of $42 \%$ and $50 \%$ above control values for corresponding lysophospholipids occurred in platelets stimulated with U46619, whereas this was blocked by the presence of GR32191B.

To demonstrate that AA was lost from the ethanolaminecontaining phospholipids upon low-level collagen exposure, mass determinations were performed (Table 4). The results indicated a significant $(P<0.05)$ net loss of AA from the plasmenylethanolamine (by $0.74 \pm 0.19 \mathrm{nmol} / 5 \times 10^{8}$ platelets) and the phosphatidylethanolamine (by $0.74 \pm 0.17 \mathrm{nmol} / 5 \times 10^{8}$ platelets) due to agonist stimulation.

\section{DISCUSSION}

As pointed out by Kambayashi et al. [11], limited information is available on the stimulus-linked metabolism of plasmenylethanolamine in platelets, owing to the relatively poor labelling of this ether phospholipid with radioactive AA, and the need to resolve the 1-alkenyl from the corresponding 1-acyl sub-class of ethanolaminephospholipid. Such labelling studies have demonstrated a marked rise in radioactive AA appearing in plasmenylethanolamine with the use of thrombin $[2,4,5,7,9]$, a strong agonist, but do not provide direct evidence of plasmenylethanolamine breakdown via phospholipase $A_{2}$. The latter findings have provided evidence for the transacylation pathway which mediates the transfer of AA from phosphatidylcholine to plasmenylethanolamine [10]. A corresponding decrease in the mass of plasmenylethanolamine has been reported [37] in human platelets exposed to thrombin, as has the accumulation of lysoplasmenylethanolamine in human [3] and rabbit [38] platelets. With low levels of collagen as an agonist (i.e. $2 \mu \mathrm{g} / \mathrm{ml}$ of platelet suspension), extreme difficulty in detecting any measurable loss of individual labelled/unlabelled phospholipids has been indicated. The present experimental approach, which employed pre-labelling of the plasmenylethanolamine (and phosphatidylethanolamine) in intact human platelets with $\left[{ }^{3} \mathrm{H}\right]$ ethanolamine, provided the opportunity to monitor the lysoplasmenylethanolamine generated via phospholipase $A_{2}$ activity using low levels of collagen (with or without $\mathrm{BW} 755 \mathrm{C}$ ) and the $\mathrm{TxA}_{2}$ mimetic (U46619) as agonists. Free $\left[{ }^{3} \mathrm{H}\right]$ ethanolamine can potentially enter ethanolamine phospholipid through a combination of base exchange [39] and biosynthetic reactions de novo $[40,41]$.

As reviewed by Arita et al. [42], collagen addition to platelets elicits various biochemical responses in addition to platelet aggregation, including inositol phosphate formation, intracellular $\mathrm{Ca}^{2+}$ mobilization, $\mathrm{AA}$ release and $\mathrm{TxA} \mathrm{A}_{2}$ generation. Collageninduced platelet responses can be distinguished by $\mathrm{TxA}_{2}$-independent and -dependent events, with the initial TxA $\mathrm{A}_{2}$-independent process involving activation of phospholipase $A_{2}$, leading to AA release and $T x A_{2}$ synthesis. The $T x A_{2}$ generated in turn activates phospholipase $A_{2}$ via transmembrane signalling processes for further AA release. The present findings indicate that collagen stimulation of human platelets activates the phospholipase $\mathrm{A}_{2}$-mediated generation of lysoplasmenylethanolamine from plasmenylethanolamine in an eicosanoid-independent manner (Figure 1 and Table 1). We observed that $72 \%$ of the generated lysoplasmenylethanolamine was maintained when eicosanoid synthesis was inhibited by the dual cyclo-oxygenase/lipoxygenase inhibitor, BW755C (Table 1). The moderate diminution in agonist-induced lysoplasmenylethanolamine accumulation observed upon BW755C addition (by $28 \%$ ) and the ability of U46619 to promote moderately (by $29 \%$ ) its generation (Table 1) indicate some hydrolysis of plasmenylethanolamine via an eicosanoid/TxA $\mathrm{A}_{2}$-mediated mechanism. As observed with BW755C, the presence of $1 \mu \mathrm{M}$ GR32191B, the TxA $\mathrm{A}_{2}$-receptor antagonist, also had only a moderate inhibitory effect on the generation of lysoplasmenylethanolamine in collagen-stimulated platelets (Table 3 ). This suggests that the eicosanoid-dependent breakdown of these phospholipids is mainly due to $\operatorname{TxA}_{2}$, the potent pro-aggregatory metabolite of $\mathrm{AA}$. The corresponding data on the agonist-induced generation of labelled lysophosphatidylethanolamine (Figure 2 and Table 1) are in agreement with previous work demonstrating the involvement of both eicosanoid-independent and -dependent mechanisms for the cleavage of phosphatidylethanolamine by phospholipase $A_{2}$ in collagen-stimulated human platelets [21]. In contrast with the finding on ethanolamine phospholipids, the activated phospholipase $\mathbf{A}_{2}$-mediated hydrolysis of phosphatidylcholine to 1acyl (2-lyso) derivative upon platelet stimulation with low levels of collagen (under the same conditions used herein) was found to be highly sensitive to inhibition by BW755C, indicating little eicosanoid-independent hydrolysis [21].

The radioactivity ratios (lysophosphatidylethanolamine/ lysoplasmenylethanolamine) in the accumulated lyosphospholipids (agonist-induced) upon collagen stimulation (+ BW755C), which were smaller than the corresponding parent phospholipids (1.2 versus 2.3; Table 2), suggests some preferential hydrolysis of the plasmenylethanolamine via the eicosanoid-independent process. Conversely, the somewhat higher ratio in the lysophospholipids relative to the parent phospholipids (3.3 versus 2.3) with U46619 as agonist (Table 2) suggests a moderate preference for phosphatidylethanolamine hydrolysis via the 
eicosanoid/TxA $\mathbf{A}_{2}$-dependent pathway. However, a firm conclusion is difficult in this regard, since these lysophospholipids are subject to some hydrolysis by lysoplasmalogenase/ lysophospholipase activities as well as acylation/transacylation reactions $[10,43]$. Studies in vitro in disrupted resting platelets have demonstrated the presence of a phospholipase $A_{2}$ which can cleave plasmenylethanolamine [44]. Although the ethanolaminecontaining phospholipid was not specifically studied, a plasmalogen-selective phospholipase $\mathrm{A}_{2}\left(\mathrm{Ca}^{2+}\right.$-independent) has been identified [45] and recently purified [46] from dog myocardium, which acts on plasmenylcholine. $\mathrm{A} \mathrm{Ca}^{2+}$-dependent phospholipase $\mathbf{A}_{2}$ which hydrolyses plasmenylcholine has been identified and purified from sheep platelets [47]. The mass measurements (Table 4) showing a significant loss of AA from plasmenylethanolamine support a simultaneous release of AA and lysoplasmenylethanolamine generation via enhanced phospholipase $A_{2}$ activity in collagen-stimulated human platelets.

The relationship of phospholipase $\mathrm{A}_{2}$-mediated hydrolysis of plasmenylethanolamine as studied herein upon collagen stimulation to platelet aggregation, phosphoinositide turnover, $\mathrm{Ca}^{2+}$ mobilization and protein kinase activation remains to be studied. Interestingly, Moriyama et al. [48] have concluded that $\mathrm{TxA}_{2}$ directly causes $\mathrm{Ca}^{2+}$ mobilization without further activation of phospholipase $\mathrm{C}$ upon collagen addition to human platelets. It is possible that the eicosanoid-independent release of AA from plasmenylethanolamine is particularly important in this regard. Also of interest is the similarity of the time course of generation of $\left[{ }^{3} \mathrm{H}\right]$ ethanolamine-containing lysophospholipids in collagenstimulated platelets (Figures 1 and 2) with that of the timedependent formation of $\mathrm{TxB}_{2}$ reported by Karniguian et al. [49].

In conclusion, the present study indicates that plasmenylethanolamine, which represents the single predominant reservoir of AA-containing phospholipid in resting human platelets, is hydrolysed in collagen-stimulated platelets to a considerable extent via eicosanoid/TxA $\mathrm{A}_{2}$-independent as well as eicosanoid/ $\mathrm{TxA}_{2}$-dependent mechanisms. The eicosanoid-independent hydrolysis of plasmenylethanolamine by phospholipase $A_{2}$ may represent an important source of releasable AA for the initial $\mathrm{TxA}_{2}$ synthesis and $\mathrm{TxA}_{2}$-induced hydrolysis of other phospholipids via phospholipase $A_{2}$ (for further AA release) and phospholipid-mediated signalling processes.

M.E.T. was a recipient of Studentships from the 'Fonds National Suisse de la Recherche Scientifique' and the 'Fondation pour l'Encouragement de la Recherche sur la Nutrition en Suisse'. We appreciate the grant support received from the Medical Research Council of Canada.

\section{REFERENCES}

1 Bills, T. K., Smith, J. B. and Silver, M. J. (1977) J. Clin. Invest. 60, 1-6

2 Rittenhouse-Simmons, S., Russell, F. A. and Deykin, D. (1977) Biochim. Biophys. Acta 488, 370-380

3 Broekman, M. J., Ward, J. W. and Marcus, A. J. (1980) J. Clin. Invest. 66, 275-283

4 Colard, 0., Breton, M. and Bereziat, G. (1986) Biochim. J. 233, 691-695

5 Purdon, A. D., Patelunas, D. and Smith, J. B. (1987) Lipids 22, 116-120

6 Hanasaki, K., Nakano, T. and Arita, H. (1987) J. Biochem. (Tokyo) 102, 5-8

7 Rittenhouse-Simmons, S., Russell, F. A. and Deykin, D. (1976) Biochem. Biophys. Res. Commun. 70, 295-301
8 Colard, 0., Breton, M. and Bereziat, G. (1984) Biochem. J. 222, 657-662

9 Purdon, A. D. and Smith, J. B. (1985) J. Biol. Chem. 260, 12700-12704

10 Kramer, R. M. and Deykin, D. (1983) J. Biol. Chem. 258, 13806-13811

11 Kambayashi, J., Kawasaki, T., Tsujinaka, T., Sakon, M., Oshiro, T. and Mori, T. (1987) Biochem. Int. 14, 241-247

12 Mahadevappa, V. G. and Holub, B. J. (1986) Biochem. Biophys. Res. Commun. 134, 1327-1333

13 Chau, L. Y. and Tai, H. H. (1981) Biochem. Biophys. Res. Commun. 100, 1588-1695

14 Siess, W., Cuatrecasas, P. and Lapetina, E. G. (1983) J. Biol. Chem. 258, 4683-4686

15 Sano, K., Takai, Y., Yamanishi, J. and Nishizuka, Y. (1983) J. Biol. Chem. 258, 2010-2013

16 Best, L. C., Holland, T. K., Jones, P. B. B. and Russell, R. G. G. (1980) Thromb. Haemostatis 43, 38-40

17 Nakano, T., Hanasaki, K. and Arita, H. (1989) J. Biol. Chem. 264, 5400-5406

18 Narita, H., Park, H. J., Tanaka, K., Matsuura, T. and Kito, M. (1985) J. Biochem. (Tokyo) 98, 1063-1068

19 Pollock, W. K., Armstrong, R. A., Brydon, L. J., Jones, R. L. and Macintyre, D. E. (1984) Biochem. J. 219, 833-842

20 Nozawa, Y., Nakashima, S. and Nagata, K. (1991) Biochim. Biophys. Acta 1002, 219-238

21 Thomas, L. M. and Holub, B. J. (1991) Biochim. Biophys. Acta 1081, 92-98

22 Mueller, H. W., Purdon, A. D., Smith, J. B. and Wykle, R. L. (1983) Lipids 18, 814-819

23 Natarajan, V., Zuzarte-Augustin, M., Schmid, H. H. O. and Graff, G. (1983) Thromb. Res. 30, 119-125

24 Takamura, H., Tanaka, K. I., Matsuura, T. and Kito, M. (1989) J. Biochem. (Tokyo) 105, 168-172

25 Aukema, H. M. and Holub, B. J. (1989) J. Lipid Res. 30, 59-64

26 Mahadevappa, V. G. and Holub, B. J. (1982) Biochim. Biophys. Acta 713, 73-79

27 Mustard, J. F., Perry, D. W., Ardlie, N. G. and Packam, M. A. (1972) Br. J. Haematol. 22, 193-204

28 Smith, J. B., Dangelmaier, C. and Mauco, G. (1985) Biochim. Biophys. Acta 835, 344-351

29 Takahara, K., Murray, R., Fitzgerald, G. A. and Fitzgerald, D. J. (1990) J. Biol. Chem. 265, 6836-6844

30 Bligh, E. G. and Dyer, W. J. (1959) Can. J. Biochem. Physiol. 37, 911-917

31 Horrocks, L. A. (1968) J. Lipid. Res. 9, 469-472

32 Vinson, J. A. and Hoyman, J. E. (1977) J. Chromatogr. 135, 226-228

33 Granström, E. and Kindhal, H. (1978) Prostaglandins Thromboxane Res. 5, 119-210

34 Holub, B. J. and Skeaff, C. M. (1987) Methods Enzymol. 141, 234-244

35 Steel, R. G. D. and Torrie, J. H. (1980) Principles and Procedures of Statistics, 2nd edn., McGraw-Hill, New York

36 El Tamer, A., Record, M., Fauvel, J., Chap, H. and Douste-Blazy, L. (1984) Biochim. Biophys. Acta 793, 213-220

37 Takamura, H., Narita, H., Park, H. J., Tanaka, K., Matsuura, T. and Kito, M. (1987) J. Biol. Chem. 262, 2262-2269

38 Morikawa, S., Taniguchi, S., Fujiwara, M., Kumada, K., Ishida, T., Hiriike, K. and Nozaki, M. (1989) Thromb. Res. 55, 427-438

39 Bjerve, K. S. (1973) Biochim. Biophys. Acta 296, 549-562

40 Mozzi, R., Gresele, P., Siepi, D., Goracci, G., Nenci, G. G. and Porcellati, G. (1987) Thromb. Res. 45, 687-693

41 Esko, J. D. and Raetz, C. R. H. (1983) Enzymes 3rd Ed. 16, 207-253

42 Arita, H., Nakano, T. and Hanasaki, K. (1989) Prog. Lipid Res. 28, 273-301

43 Jurkowitz-Alexander, M., Ebata, H., Mills, J. S., Murphy, E. J. and Horrocks, L. A (1989) Biochim. Biophys. Acta 1002, 203-212

44 Colard, O., Breton, M. and Bereziat, G. (1987) Biochim. Biophys. Acta 921, 333-340

45 Wolf, R. A. and Gross, R. W. (1985) J. Biol. Chem. 260, 7295-7303

46 Hazen, S. L., Stuppy, E. J. and Gross, R. W. (1990) J. Biol. Chem. 265, 10622-10630

47 Loeb, L. A. and Gross, R. W. (1986) J. Biol. Chem. 261, 10467-10470

48 Moriyama, T., Takamura, H., Narita, H., Tanaka, K. I., Matsuura, T. and Kito, M. (1988) J. Biochem. (Tokyo) 103, 901-902

49 Karniguian, A., Grelac, F., Levy-Toledano, S., Legrand, Y. J. and Rendu, F. (1990) Biochem. J. 268, 325-331 